\title{
Ocorrência de Salmonella spp. em Cebus spp. mantidos em Centros de Triagem de Animais Silvestres no Nordeste do Brasil
}

\author{
Débora Rochelly Alves Ferreira ${ }^{1 *}$ \\ André de Souza Santos ${ }^{1}$ \\ Paulo Guilherme Carniel Wagner ${ }^{2}$ \\ Eliane Moura Falavina dos Reis ${ }^{3}$ \\ José Wilton Pinheiro Júnior ${ }^{1}$ \\ Wagnner José Nascimento Porto ${ }^{4}$ \\ Leonildo Bento Galiza da Silva ${ }^{1}$ \\ Eneida Willcox Rego ${ }^{1}$ \\ Rinaldo Aparecido Mota ${ }^{1}$

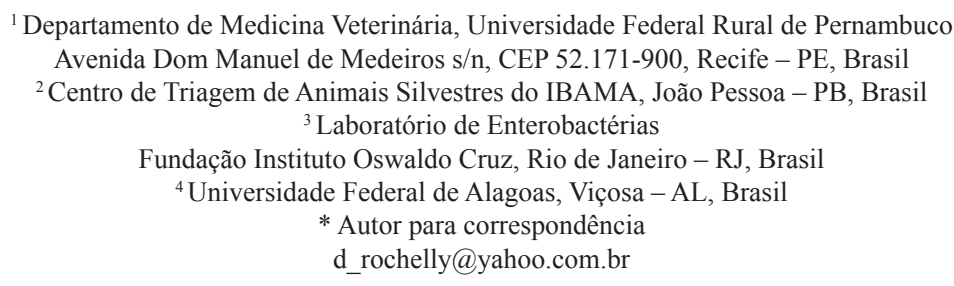

Submetido em 11/08/2011

Aceito para publicação em 27/12/2011

\section{Resumo}

Objetivou-se com este estudo isolar e identificar os sorovares de Salmonella spp. em Cebus spp. mantidos em cativeiro. Foram investigados 28 animais mantidos em Centros de Triagem de Animais Silvestres (CETAS) do Nordeste do Brasil. As amostras foram obtidas com auxílio de suabes introduzidos no reto dos animais, sendo posteriormente processados para o isolamento da bactéria em meios seletivos seguido da identificação dos sorovares por meio de provas bioquímicas. Das 28 amostras analisadas, cinco $(17,9 \%)$ foram positivas para Salmonella spp. e os sorovares identificados foram Infantis (40\%), Panama (40\%) e Newport (20\%). Ocorreu associação significativa $(\mathrm{p}<0,05)$ para as variáveis: instituição $(\mathrm{p}=0,026)$, armazenamento de frutas, verduras e hortaliças $(\mathrm{p}=0,026)$, fornecimento de carne crua $(\mathrm{p}=0,026)$, tipo de bebedouro $(\mathrm{p}=0,026)$ e higienização diária de bebedouros $(\mathrm{p}=0,026)$. Este estudo demonstrou que infecções por Salmonella spp. ocorrem em populações de Cebus mantidos em cativeiro e apontou para a necessidade de se realizar investigações periódicas em todo o plantel, assim como implantar medidas de controle para evitar riscos tanto à saúde dos animais como à saúde pública.

Palavras-chave: Diagnóstico; Enterobactérias; Primatas; Salmonelose 


\section{Abstract}

Occurrence of Salmonella spp. in Cebus spp. maintained in Wild Animals Sorting Centers in the Northeastern Brazil. The aim of this study was to isolate and identify the serovars of Salmonella spp. in Cebus spp. kept in captivity. Twenty eight animals maintained in Wild Animal Sorting Centers (CETAS) were investigated in the Northeastern Brazil. The samples were obtained with the aid of swabs introduced in the rectum of the animals, which were afterwards processed to isolate the bacterium in selective media, and then the serovars were identified through biochemical testing. Out of 28 samples analyzed, $5(17.9 \%)$ were positive for Salmonella spp. and the identified serovars were Infantis (40\%), Panama (40\%), and Newport (20\%). There was a significant association $(\mathrm{p}<0.05)$ for the variables: institution $(\mathrm{p}=0.026)$, fruit and vegetable stocking $(\mathrm{p}=0.026)$, raw meat supply $(\mathrm{p}=0.026)$, type of drinking fountains $(\mathrm{p}=0.026)$, and daily cleaning of drinking fountains ( $\mathrm{p}=0.026$ ). This study showed that infections caused by Salmonella spp. occur in Cebus populations kept in captivity and it pointed out to the need for carrying out periodic investigations in all the breeding stok, as well implementing control measures to avoid risks both to animal's health and public health.

Key words: Diagnosis; Enterobacteria; Primates; Salmonellosis

\section{Introdução}

Os macacos-prego (Cebus) são primatas neotropicais de médio porte com alta capacidade cognitiva; são onívoros e na natureza se alimentam principalmente de frutas, insetos e pequenos vertebrados (FRAGASZY et al., 2004). No cativeiro, os alimentos utilizados geralmente são frutas, verduras, produtos de origem animal e rações (em alguns centros especializadas para primatas, enquanto em outros ração para cães).

As doenças de origem alimentar podem ser provocadas por diversos grupos de microrganismos, incluindo bactérias do gênero Salmonella. As principais vias de transmissão são ingestão de produtos de origem animal mal cozidos e fontes de água contaminadas com fezes de animais infectados (MACIEL et al., 2004).

Infecção por Salmonella spp. tem sido descrita em humanos (MERMIN et al., 2004; CHEN et al., 2005), alimentos (FUNK, 2003; CASTAGNA et al., 2005; LIMA et al., 2009; LOUREIRO et al., 2010; SILVA et al., 2010a), animais domésticos (MORSE et al., 1976; FEDORKA-CRAY et al., 1998; MACIEL et al., 2004; RIBEIRO et al., 2010) e diversas espécies de animais silvestres (GOPEE et al., 2000; MERMIN et al., 2004; JIJÓN et al., 2007; COLEGROVE et al., 2010; NUNES et al., 2010; SILVA et al., 2010b; SOUSA et al., 2010), incluindo relatos em primatas (GOOD et al., 1969; GIANNELLA et al., 1977; SCHIEFER; LOEW, 1978; TANAKA; KATSUBE, 1981; TRIBE; FLEMING, 1983; SOUZA JÚNIOR et al., 2008; KNÖBL et al., 2011).
A realização de inquéritos que demonstrem a infecção por enterobactérias em populações de primatas mantidos em cativeiro é de grande importância (SOUZA JÚNIOR et al., 2008) já que bactérias do gênero Salmonella estão entre os patógenos de importância em saúde pública e animal e acometem colônias de primatas (YASUTOMI, 2010) e humanos (TANAKA; KATSUBE, 1981). Desta forma, objetivou-se isolar e identificar os sorovares de Salmonella spp. em Cebus spp. mantidos em Centros de Triagem de Animais Silvestres do Instituto Brasileiro do Meio Ambiente e Recursos Renováveis CETAS/IBAMA.

\section{Materiais e Métodos}

Foram coletadas amostras de 28 primatas de macacos do gênero Cebus de diferentes espécies, sexos e idades, procedentes do CETAS/IBAMA de Alagoas (09³9'57'S e 35 44'07'O) e do CETAS/IBAMA da Paraíba (0706’54’S e 3451'47’O). As amostras foram coletadas no período de fevereiro a setembro de 2010. Utilizou-se amostragem não probabilística por conveniência e foram amostrados os animais de acordo com a facilidade de captura.

Para a coleta das amostras os animais foram contidos de forma física utilizando-se puçá e luvas de couro, seguida de contenção química utilizandose associação de cloridrato de cetamina na dose de $10 \mathrm{mg} / \mathrm{kg}$, cloridrato de xilazina na dose de $1 \mathrm{mg} / \mathrm{kg}$ e 
diazepam na dose de $1 \mathrm{mg} / \mathrm{kg}$ administrados por via intramuscular. Os animais foram submetidos ao exame clínico, sendo empregados meios semiológicos como palpação, inspeção direta e indireta (DINIZ, 1997). Para cada animal foi preenchida uma ficha de identificação individual padrão do Centro de Proteção de Primatas Brasileiros contendo a identificação do CETAS.

As amostras foram coletadas por meio de suabes estéreis introduzidos no reto de cada animal. Após a coleta, o material foi mantido sob refrigeração em caixa isotérmica e encaminhado ao Laboratório de Doenças Infecciosas dos Animais Domésticos da Universidade Federal Rural de Pernambuco.

As amostras foram pré-enriquecidas em água peptonada tamponada a $1 \%$ e incubadas a $37^{\circ} \mathrm{C}$ por $24 \mathrm{~h}$. Posteriormente, transferiu-se da solução original, $1 \mathrm{~mL}$ para os caldos Selenito-Cistina (Oxoide $\AA$ ) e RappaportVassiliadis $\left(\right.$ Oxoide ${ }^{\circledR}$ ) acrescido de Novobiocina a $4 \%$ (SIGMA $\AA$ ) que foram incubados a $37^{\circ} \mathrm{C}$ por $24 \mathrm{~h}$. A partir dos caldos de enriquecimento as amostras foram semeadas em placas contendo os meios ágar Xilose Lisina Desoxicolato (XLD) (Oxoide $\left.{ }^{\circledR}\right)$ e ágar Verde-brilhante incubados a $37^{\circ} \mathrm{C}$ por $24 \mathrm{~h}$. As colônias suspeitas foram submetidas às provas bioquímicas Ágar Tríplice Açúcar Ferro (TSI) (Oxoide $\left.{ }^{\circledR}\right)$, Ágar lisina-ferro (LIA) (Oxoide $\AA$ ), caldo ureia (Oxoide $\left.{ }^{\circledR}\right)$ (COSTA; HOFER, 1972) e incubadas a $37^{\circ} \mathrm{C}$ por $24 \mathrm{~h}$ Após a identificação presuntiva, as amostras suspeitas foram encaminhadas em ágar nutriente (Oxoide $\left.{ }^{\circledR}\right)$ para o Laboratório de Enterobactérias da Fundação Instituto Oswaldo Cruz (FIOCRUZ), Rio de Janeiro - RJ para confirmação do gênero e tipificação do sorotipo (LE MINOR; POPOFF, 1987).

A análise estatística para verificar a associação entre as variáveis - instituição, sexo e idade - foi realizada pelo teste Exato de Fisher. Foi utilizado para a execução dos cálculos estatísticos o programa Epi-Info (DEAN et al., 1994).

A pesquisa foi autorizada pelo Instituto Chico Mendes de Conservação da Biodiversidade ICMBio/ SISBIO, número 16218-1/2008 e pelo Comitê de Ética da Universidade Federal de Campina Grande (UFCG), protocolo número 105/2009.

\section{Resultados}

Das 28 amostras analisadas, cinco (17,9\%) foram positivas no exame microbiológico para Salmonella spp. Na identificação dos sorovares, observaram-se duas amostras Infantis (40,0\%), duas Panama (40,0\%) e uma Newport (20,0\%).

A frequência de infecção por Salmonella spp. observada por Instituição foi de 44,4\% (4/9) no CETAS/ IBAMA de Alagoas e 5,3\% (1/19) no CETAS/IBAMA da Paraíba. Quanto às espécies, observou-se que Cebus libidinosus apresentou frequência de 18,18\% (4/22) e Cebus flavius, 33,3\% (1/3). Ao avaliar a infecção por Salmonella spp. quanto ao sexo, observou-se frequência de $14,3 \%$ para machos $(3 / 21)$ e $28,6 \%$ para fêmeas $(2 / 7)$. Em relação à idade $10,0 \%$ eram animais jovens (1/10) e $26,7 \%$ adultos (4/15). Todos os animais positivos não apresentavam sinais clínicos da infecção.

Observou-se associação significativa $(\mathrm{p}<0.05)$ para as variáveis: Instituição $(\mathrm{p}=0,026)$, fornecimento de carne crua ( $p=0,026)$, armazenamento de frutas, verduras e hortaliças $(p=0,026)$, tipo de bebedouro $(p=0,026)$ e higienização diária dos bebedouros $(\mathrm{p}=0,026)$.

\section{Discussão}

Este é o primeiro registro de ocorrência de infecção por Salmonella spp. em primatas do gênero Cebus libidinosus mantidos em Centros de Triagem de Animais Silvestres nos estados de Alagoas e Paraíba, bem como em Cebus flavius, espécie recentemente redescoberta e criticamente ameaçada de extinção.

Ressalta-se a importância desses resultados do ponto de vista epidemiológico para as espécies de Cebus spp. mantidas nesses centros, pois um surto de salmonelose nesses animais poderia comprometer seriamente a saúde dos mesmos, uma vez que dependendo do sorotipo envolvido, dose infectante, idade do animal e resposta imune à infecção, a doença pode ser desencadeada com evolução para a morte.

Os sorovares identificados nesse estudo causam doença em humanos e animais através do consumo de alimentos contaminados como: carne bovina, aves e ovos 
crus (SWAMINATHAN et al., 2006; CASTILLO et al., 2008; SHINOHARA et al., 2008; SKOV et al., 2008), alimentos comumente utilizados na dieta de primatas mantidos em cativeiro.

A similaridade dos sorovares identificados em animais desse estudo com aqueles observados em casos de salmonelose em humanos tem sido descrita na literatura (RIBEIRO et al., 2010).

A ocorrência de infecções por Salmonella Infantis, Panama e Newport em C. libidinosus e C. flavius reforçam a necessidade de utilização de Equipamentos de Proteção Individual por manejadores bem como a implantação de programas de educação continuada em saúde nas instituições. De acordo com Loureiro et al. (2010), o conhecimento da distribuição geográfica dos diferentes sorovares do gênero Salmonella é importante para identificar sua incidência/prevalência e o risco à saúde da população que mantém contato com esses animais.

A identificação de animais sem sinais clínicos da salmonelose nesse estudo também alerta para o estado de portador saudável que é um fator relevante na transmissão de Salmonella e favorece a manutenção da cadeia de transmissão da salmonelose (WEILL, 2008). Souza Júnior et al. (2008) também diagnosticaram infecção assintomática por Salmonella spp. em primatas mantidos em cativeiro, corroborando os achados obtidos nesse estudo.

$\mathrm{Na}$ análise de associação observou-se diferença significativa entre o isolamento de Salmonella spp. e a variável Instituição. Isso pode ser causado por diferenças no manejo sanitário que cada Instituição preconiza, fato que contribui significativamente para ausência ou presença de infecção nos animais.

O fornecimento de produtos de origem animal crus na dieta dos primatas mantidos em cativeiro é uma prática que deve ser excluída do manejo alimentar dos plantéis estudados para evitar infecção por Salmonella spp. já que trabalhos reportam a presença do referido patógeno em diversos produtos de origem animal (TAN; SHELEF, 1999; LIMA et al., 2009; BRITO et al., 2010; VILELA et al., 2010).

De acordo com Wierup e Häggblom (2010), efetivos procedimentos de Análise de Perigos e Pontos
Críticos de Controle (APPCC) devem ser implantados baseados no controle e em ações corretivas para redução da contaminação alimentar por Salmonella spp. Esses procedimentos devem ser utilizados pelos centros estudados, já que se diagnosticou infecção por Salmonella e pelo fato desse patógeno ser transmitido por alimentos contaminados (WEIR et al., 2004; CHEN et al., 2005; MILER et al., 2010).

Balian et al. (2006) reforçaram que a adoção de boas práticas, quando monitoradas pode ser economicamente viável para reduzir a presença e ou circulação de microrganismos como Salmonella spp. em setores de manipulação e preparo de alimentos em zoológicos, bem como, pode oferecer condições para redução dos gastos com diagnóstico, tratamento e agravos à saúde animal. Desta forma, a higienização de frutas e verduras, bem como a aquisição de produtos de origem animal de boa qualidade e inspecionados são medidas importantes para o controle de patógenos de veiculação alimentar, como é o caso da Salmonella.

A ocorrência de associação entre infecção por Salmonella spp. em Cebus spp. e utilização de bebedouros tipo vasilha observada neste estudo reforça a possibilidade de animais infectados defecarem e contaminarem a água, sugerindo a substituição dos bebedouros tipo vasilha por bebedouros tipo chupeta. Geralmente essa água fica imprópria para o consumo em cativeiro pela dificuldade de manter bebedouros íntegros e com água limpa, já que os recipientes são usados nas diversas atividades manipulativas realizadas pelos macacos ou pelo comportamento destes em lavar certos alimentos antes de consumi-los (LAROQUE et al., 2009). A água usada na dessedentação de animais pode representar risco em relação à salmonelose (SOUZA et al., 1992).

A manutenção ilegal de animais silvestres em cativeiro é um risco à saúde pública, já que o conhecimento sobre zoonoses, dentre elas a salmonelose é restrito (NUNES et al., 2010). Primatas mantidos nos CETAS normalmente são procedentes do tráfico de animais silvestres e na maioria das vezes no cativeiro domiciliar esses animais são avaliados por médicos veterinários apenas em situações de emergência. A falta de manejo sanitário adequado facilita a disseminação de patógenos para outros animais e humanos. 
Além da investigação de saúde nos animais é importante uma avaliação sistemática nos funcionários, pois de acordo com Ciffoni et al. (2007), alterações no estado sanitário de funcionários como problemas gastrintestinais podem comprometer a saúde dos animais e de outros profissionais.

Infecções por Salmonella spp. ocorrem em populações de Cebus mantidos em cativeiro e apontam a necessidade de se realizar investigações periódicas em todo o plantel, assim como implantar medidas de controle para evitar riscos à saúde dos animais e pública.

\section{Agradecimentos}

À CAPES pela concessão da bolsa de estudos. Aos CETAS/IBAMA de Alagoas e da Paraíba por permitirem a coleta do material biológico.

\section{Referências}

BALIAN, S. C.; TELLES, E. O.; GOMES, M. S.; AZEVEDO, V. L.; SANCHEZ, A. S.; SOUZA, O. B.; GRESPAN, A.; FERREIRA, A. J. P.; PINHEIRO, S. R. Boas práticas de preparação de alimentos em um zoológico do estado de São Paulo; planejamento, implementação e levantamento de custos. Veterinária e Zootecnia, Botucatu, v. 13, n. 2, p. 208-218, 2006.

BRITO, D. A. P.; ALVES, L. M. C.; COSTA, F. N. Detecção de Salmonella albany, Staphylococcus coagulase positivos e microorganismos mesófilos em carcaças de frango in natura. Arquivo do Instituto Biológico de São Paulo, São Paulo, v. 77, n. 1, p. 149-152, 2010

CASTAGNA, S. M. F.; MULlER, M.; MACAGNA, M.; RODENBUSCHE, C. R.; CANAL, C. W.; CARDOSO, M. Detection of Salmonella sp. from porcine origin: a comparison between a pcr method and standard microbiological techniques. Brazilian Journal of Microbiology, São Paulo, v. 36, p. 373-377, 2005.

CASTILlO, A. C. G.; MARTíNEZ, L. H. P.; APODACA, N. L. C. Salmonelosis y campilobacteriosis, las zoonosis emergentes de mayor expansión en el mundo. Veterinária México, México, v. 39, n. 1, p. 81-90, 2008.

CHEN, T.; THIEN, P.; LIAW, S.; FUNG, C.; SIU, L. K. First report of Salmonella enterica Serotype Panama meningitis associated with consumption of contaminated breast milk by a neonate. Journal of Clinical Microbiology, Washington, v. 43, n. 10, p. 5400-5402, 2005.

CIFFONI, E. M. G.; ARNS, G. C.; PACHALY, J. R. Biossegurança e riscos biológicos nos setores cirúrgicos das unidades veterinárias de parques zoológicos. A Hora Veterinária, São Paulo, n. 156, p. 41-46, 2007.
COLEGROVE, K. M.; LEGER, J. A. ST.; RAVERTY, S.; JANG, S.; BERMAN-KOWALEWSKI, M.; GAYDOS, J. K. Salmonella newport omphaloarteritis in a stranded killer whale (Orcinus orca) neonate. Journal of Wildlife Diseases, Atlanta, v. 46, n. 4, p. 13001304, 2010

COSTA, E.; HOFER, G. A. Investigação sobre a ocorrência de Salmonella em esgotos sanitários da cidade do Rio de Janeiro. Memórias do Instituto Oswaldo Cruz, Rio de Janeiro, v. 70, p. 221-236, 1972

DEAN, A. G.; DEAN, J. A.; COULOMERIER, D.; BRENDEL, K. A.; SMITH, D. C.; BURTON, A. H.; DICKER, R. C.; SULIVAN, K. M.; FAGAN, R. F.; ARNER, T. G. Epi Info, Version 6: a word processing, data bases, and statistic program for epidemiology on microcomputers. Atlanta: Centers for Disease Control and Prevention, 1994. S/ paginação.

DINIZ, L. S. M. Primatas em cativeiro. Manejo e problemas veterinários. São Paulo: Ícone, 1997. 197 p.

FEDORKA-CRAY, P. J.; DARGATZ, D. A.; THOMAS, L. A.; GRAY, J. T. Survey of Salmonella serotypes in feedlot cattle. Journal of Food Protection, Des Moines, v. 61, n. 5. p. 525-530, 1998.

FRAGASZY, D. M.; VISALBERGHI, E., FEDINGAN, L. M. The complete capuchin: the biology of the Genus Cebus. Massachusetts: University of Cambridge Press, 2004. 339 p.

FUNK, J. Pre-harvest food safety diagnostics for Salmonella serovars. Part 1: Microbiological culture. Journal of Swine Health and Production, Perry, v. 11, n. 2, p. 87-90, 2003

GIANNELLA, R. A.; ROUT, W. R.; FORMAL, S. B. Effect of indomethacin on intestinal water transport in Salmonella-infected rhesus monkeys. Infection and Immunity, Washington, v. 17, n. 1, p. 136-139, 1977.

GOOD, R. C.; MAY, B. D.; KAWATOMARI, T. Enteric pathogens in monkeys. Journal of Bacteriology, Washington, v. 97, n. 3. p. 1048-1055, 1969.

GOPEE, N. V.; ADESIYUN, A. A.; CAESAR, K. Retrospective and longitudinal study of salmonellosis in captive wildlife in Trinidad. Journal of Wildlife Diseases, Atlanta, n. 36, v. 2, p. 284-293, 2000.

JIJÓN, S.; WETZEL, A.; LeJEUNE, J. Salmonella enterica isolated from wildlife at two ohio rehabilitation centers. Journal of Zoo and Wildlife Medicine, Yulee, v. 38, n. 3, p. 409-413, 2007.

KNÖBL, T.; ROCHA, L. T.; MENÃO, M. C.; IGAYARAS, C. A. S.; PAIXÃO, R.; MORENO, A. M. Salmonella Yoruba infection in white-tufted-ear marmoset (Callitrhrix jacchus). Pesquisa Veterinária Brasileira, Rio de Janeiro, v. 31, n. 8, p. 707-710, 2011.

LAROQUE, P. O.; WAGNER, P. G. C.; SENNA, M. B.; FERREIRA, D. R. A.; ANDRADE, L. H. M.; SILVA, T. C. F. Bebedouro do tipo chupeta para macaco-prego gênero Cebus Erxleben (1777), Primates Cebidae. In: ENCONTRO INTERNACIONAL DE MEDICINA DA CONSERVAÇÃO, 2, 2009, Recife, Resumos... Recife: Instituto Brasileiro para a Medicina da Conservação, 2009. Versão eletrônica.

LE MINOR, L.; POPOFF, M. Y. Request for an opinion. Designation of Salmonella enterica sp. nov., nom. rev., as the type and only species of the genus Salmonella. International Journal of Systematic Bacteriology, Washington, v. 37, p. 465-468, 1987. 
LIMA, E. T.; ANDREATTI FILHO, R. L.; PINTO, J. P. A. N. Perfil de suscetibilidade antimicrobiana de sorotipos de Salmonella isolados de produtos avícolas. Veterinária e Zootecnia, Botucatu, v. 16, n. 2 , p. $394-400,2009$

LOUREIRO, E. C. B.; MARQUES, N. D. B.; RAMOS, F. L. P.; REIS, E. M. F.; RODRIGUES, D.; HOFER, E. Sorovares de Salmonella de origem humana identificados no Estado do Pará, Brasil, no período de 1991 a 2008. Revista Pan-Amazônica de Saúde, Ananindeua, v. 1, n. 1, p. 93-100, 2010.

MACIEL, B. M.; ARGÔLO FILHO, R. C.; FREITAS, E. S.; KRUSCHEWSKY, F. F.; SANTOS, B. F.; ROCHA, G. D.; WETLER, R. M. C.; MARTINS, L. A. F. Ocorrência de sorotipos exóticos de Salmonella encontrados em cães assintomáticos nos distritos do município de Ilhéus/BA - Brasil. Brazilian Journal of Veterinary Research Animal Science, São Paulo, v. 41, p. 247253, 2004.

MERMIN, J.; HUTWAGNER, L.; VUGIA, D.; SHALLOW, S.; DAILY, P.; BENDER, J.; KOEHLER, J.; MARCUS, R.; ÂNGULO, F. J. Reptiles, Amphibians, and Human Salmonella infection: a population-based, case-control study. Clinical Infectious Diseases, Oxford, v. 38. n. 3, p. 253-261, 2004.

MILLER, T.; PRAGER, R.; RABSH, W.; FEHLHABER, K.; VOSS, M. Epidemiological relationship between Salmonella Infantis isolates of human and broiler origin. Lohomann Information, Cuxhaven, v. 45, n. 2, p. 27-31, 2010.

MORSE, E. V.; DUNCAN, M. A.; ESTEP, D. A.; RIGGS, W. A.; BLACKBURN, B. O. Canine salmonellosis: a review and report of dog to child transmission of Salmonella enteritidis. American Journal of Public Health, Boston, v. 66, n. 1, p. 82-84, 1976.

NUNES, O. C.; OLIVEIRA, E. D.; LABORDA, S. S.; HOHLENWERGER, J. C.; MORAES NETO, M.; FRANKE, C. R. Isolamento e identificação de Salmonella sp. de jabutis-piranga (Chelonoidis carbonaria) oriundos do tráfico de animais silvestres. Ciência Animal Brasileira, Goiânia, v. 11, n. 1, p. 168-173, 2010.

RIBEIRO, M. G.; FERNANDES, M. C.; PAES, A. C.; SIQUEIRA, A. K.; PINTO, J. P. A. N.; BORGES, A. S. Caracterização de sorotipos em linhagens do gênero Salmonella isoladas de diferentes afecções em animais domésticos. Pesquisa Veterinária Brasileira, Seropédica, v. 30, n. 2, p. 155-160, 2010.

SCHIEFER, B.; LOEW, F. M. Amebiasis and Salmonellosis in a Woolly Monkey (Lagothrix). Veterinary Pathology, Seattle, v. 15, p. 428-431, 1978.

SHINOHARA, N. K. S.; BARROS, V. B.; JIMENEZ, S. M. C.; MACHADO, E. C. L.; DUTRA, R. A. F.; LIMA FILHO, J. L. I. V. Salmonella spp. importante agente patogênico veiculado em alimentos. Ciência e Saúde Coletiva, Rio de Janeiro, v. 13, n. 5, p. 1675-1683, 2008.

SilVA, E. P. S.; BERGAMINE, A. M. M.; OLIVEIRA, M. A. Alimentos e agentes etiológicos envolvidos em toxinfecções na região de Ribeirão Preto, SP, Brasil - 2005 a 2008 Foods and etiologic agents involved in foodborne diseases in the region of Ribeirão Preto city, State of São Paulo, Brazil - 2005 to 2008. Bepa, São Paulo, v. 7, n. 77, p. 4-10, 2010a.

SILVA, M. A.; MARVULO, M. F. V.; MOTA, R. A.; SILVA, J. C. R. A importância da ordem Ciconiiformes na cadeia epidemiológica de
Salmonella spp. para a saúde pública e a conservação da diversidade biológica. Pesquisa Veterinária Brasileira, Seropédica, v. 30. n. 7 , p. 573-580, 2010b.

SKOV, M. N.; MADSEN, J. J.; RAHBEK, C.; LODAHL, J.; JESPERSEN, J.; JORGENSEN, J.; DIETZ, H.; CHRIEL, M.; BAGGESEN, D. Transmission of Salmonella between wildlife and meat-production animals in Denmark. Journal of Applied Microbiology, Malden, v. 105, p. 1558-1568, 2008.

SOUSA, E.; BERCHIERI JUNIOR, A.; PINTO, A. A.; MACHADO, R. Z.; CARRASCO, A. O. T.; MARCIANO, J. A.; WERTHER, K. Prevalence of Salmonella spp. antibodies to Toxoplasma gondii, and Newcastle Disease in feral pigeons (Columba livia) in the city of Jaboticabal, Brazil. Journal of Zôo and Wildlife Medicine, Yulee, v. 41, p. 603-607, 2010.

SOUZA, L.C.; IARIA, S.T.; PAIN, G.V. Salmonelas e coliformes fecais em águas de bebida para animais. Revista de Saúde Pública, São Paulo, v. 26, n. 5, p. 321-327, 1992.

SOUZA JÚNIOR, J.C.; HIRANO, Z.M.B.; ÁVILAPIRES, F.D.; VIEIRA, G.R.T. Nota sobre infecção por Salmonella sp. e Shigella sp. em bugios-ruivos, Alouatta clamitans Cabrera, 1940 (Primates: Atelidae) mantidos em cativeiro. Brazilian Journal of Veterinary Research Animal Science, São Paulo, v. 45, n. 4, p. 266-268, 2008. SWAMINATHAN, B.; GERNER-SMIDT, P.; BARRETT, T. Foodborne disease trends and reports. Foodborne Pathogens and Disease, New Rochelle, v. 3, n. 2, p. 154-156, 2006.

TAN, W.; SHELEF, L. A. Automated detection of Salmonella spp. in foods. Journal of Microbiological Methods, Amsterdam, v. 37, p. 87-91, 1999.

TANAKA, Y.; KATSUBE, Y. Salmonella carriers in the imported cynomolgus monkey Macaca fascicularis. Japanese Journal Veterinary Science, Tokyo, v. 43, p. 787-789, 1981.

TRIBE, G. W.; FLEMING, M. P. Biphasic enteritis in imported cynomolgus (Macaca fascicuJaris) monkeys infected with Shige/ Ja, Salmonella and Campylobacter species. Laboratory Animals, London, v. 17, p. 65-69, 1983.

VILELA, S. M. O.; FAGUNDES, D. L.; PINHEIRO JUNIOR, J. W.; SUKAS, T. N.; MOTA, R. A. Pesquisa de Salmonella spp. e Staphylococus spp., coliformes totais e termotolerantes em carne de avestruz (Struthio camelus) industrialmente processada. Veterinária e Zootecnia, Botucatu, v. 17, n. 3, p. 354-358, 2010.

WEILL, F. X. Salmonella: Épidémiologie, typage et résistence aux antibiotiques. Revue Francophone des Laboratoires, Netherlands, n. 400, p. 37-47, 2008.

WEIR, E.; DORÉ, K.; CURRIE, A. Enhanced surveillance for Salmonella Newport. Canadian Medical Association or its Licensors, Ottawa, v. 171, n. 2, p. 127-128, 2004.

WIERUP, M.; HÄGGBLOM, P. An assessment of soybeans and other vegetable proteins as source of salmonella contamination in pig production. Acta Veterinaria Scandinavica, Copenhagen, v. 52, n. 15, p. 1-9, 2010

YASUTOMI, Y. Establishment of specific pathogen-free macaque colonies in Tsukuba Primate Research Center of Japan for AIDS research. Vaccine, Kidlinton, v. 28S, p. B75-B77, 2010. 